University of Wollongong

Research Online

Faculty of Engineering and Information

Faculty of Engineering and Information

Sciences - Papers: Part A

Sciences

$1-1-2013$

\title{
A three-phase power flow approach for integrated 3-wire MV and 4-wire multigrounded LV networks with rooftop Solar PV
}

Md J E Alam

University of Wollongong, mjea982@uowmail.edu.au

K M. Muttaqi

University of Wollongong, kashem@uow.edu.au

Darmawan Sutanto

University of Wollongong, soetanto@uow.edu.au

Follow this and additional works at: https://ro.uow.edu.au/eispapers

Part of the Engineering Commons, and the Science and Technology Studies Commons

Research Online is the open access institutional repository for the University of Wollongong. For further information contact the UOW Library: research-pubs@uow.edu.au 


\title{
A three-phase power flow approach for integrated 3-wire MV and 4-wire multigrounded LV networks with rooftop Solar PV
}

\author{
Abstract \\ With increasing level of rooftop solar photovoltaic (PV) penetration into low voltage (LV) distribution \\ networks, analysis with realistic network models is necessary for adequate capturing of network behavior. \\ Traditional three-phase 3-wire power flow approach lacks the capability of exact analysis of 4-wire \\ multigrounded LV networks due to the approximation of merging the neutral wire admittance into the \\ phase wire admittances. Such an approximation may not be desirable when neutral wire and grounding \\ effects need to be assessed, especially in the presence of single-phase solar power injection that may \\ cause a significant level of network unbalance. This paper proposes a three-phase power flow approach \\ for distribution networks while preserving the original 3-wire and 4-wire configurations for more accurate \\ estimation of rooftop PV impacts on different phases and neutrals. A three-phase transformer model is \\ developed to interface between the 3-wire medium voltage (MV) and the 4-wire LV networks. Also an \\ integrated network model is developed for an explicit representation of different phases, neutral wires and \\ groundings of a distribution system. A series of power flow calculations have been performed using the \\ proposed approach to investigate the impacts of single-phase variable PV generation on an Australian \\ distribution system and results are presented.
}

\section{Keywords}

approach, flow, power, solar, phase, networks, three, pv, Iv, multigrounded, 4, rooftop, mv, wire, 3, integrated

Disciplines

Engineering | Science and Technology Studies

\section{Publication Details}

M. Alam, K. M. Muttaqi \& D. Sutanto, "A three-phase power flow approach for integrated 3-wire MV and 4-wire multigrounded LV networks with rooftop Solar PV," IEEE Transactions on Power Systems, vol. 28, (2) pp. 1728-1737, 2013. 


\title{
A Three-Phase Power Flow Approach for Integrated 3-Wire MV and 4-Wire Multigrounded LV Networks with Rooftop Solar PV
}

\author{
M J E Alam, Student Member, IEEE, K M Muttaqi, Senior Member, IEEE, and D Sutanto, Senior Member, IEEE
}

\begin{abstract}
With increasing level of rooftop solar PV penetration into LV distribution networks, analysis with realistic network models is necessary for adequate capturing of network behavior. Traditional three-phase 3-wire power flow approach lacks the capability of exact analysis of 4-wire multigrounded $\mathrm{LV}$ networks due to the approximation of merging the neutral wire admittance into the phase wire admittances. Such an approximation may not be desirable when neutral wire and grounding effects need to be assessed, especially in the presence of single-phase solar power injection that may cause a significant level of network unbalance. This paper proposes a three-phase power flow approach for distribution networks while preserving the original 3-wire and 4-wire configurations for more accurate estimation of rooftop PV impacts on different phases and neutrals. A three-phase transformer model is developed to interface between the 3-wire MV and the 4-wire LV networks. Also an integrated network model is developed for an explicit representation of different phases, neutral wires and groundings of a distribution system. A series of power flow calculations have been performed using the proposed approach to investigate the impacts of single-phase variable PV generation on an Australian distribution system and results are presented.
\end{abstract}

Index Terms-Thee-phase distribution networks, 3-wire and 4-wire multigrounded systems, Three-phase power flow, Rooftop solar PV impacts.

\section{INTRODUCTION}

Integration of solar photovoltaic (PV) resources in high penetration level may introduce a multitude of adverse impacts [1], [2] on distribution networks. Voltage rise [3], reverse power flow [4], voltage unbalance [2] can be listed as some of the major impacts. Analysis of network behavior under PV penetration is essential for understanding these impacts. Three phase power flow is often deployed as a tool for the analysis of network asymmetry and load imbalance in distribution networks. With an increasing level of single phase rooftop solar PV penetration, phase domain analysis is essential not only for incorporating the load unbalance and network asymmetries, but also to assess the impacts of single-phase solar PV units installed in the three phase distribution networks. Without the direct control of distribution utilities over the sizes and locations of customer-installed rooftop PV units,

This work is supported by the Australian Research Council (ARC) and Essential Energy Linkage Grant, LP100100618.

M J E Alam, K M Muttaqi and D Sutanto is with the Endeavour Energy Power Quality and Reliability Research Centre, School of Electrical, Computer, and Telecommunications Engineering, University of Wollongong, NSW 2522, Australia (e-mail: mjea982@uowmail.edu.au, kashem@uow.edu.au, soetanto@uow.edu.au)

Manuscript received. unbalanced allocation of PV resources will produce different level of impacts on different phases of a feeder, such as unbalanced impacts of voltage rise [3] and reverse power flow [4]. Unbalanced operation of networks can also affect induction motors, power electronic converters [5], and, may also decrease network voltage stability margins [6].

Medium voltage (MV) segments of distribution networks are typically constructed with 3-wire configuration whereas low voltage (LV) segments are constructed with 4-wire configuration, where the fourth wire is the neutral wire which is grounded at multiple locations along the feeder. Using the assumption of zero neutral voltage with a perfectly grounded neutral in the LV part, matrix reduction technique is typically applied to reduce the $4 \times 4$ admittance matrices corresponding to LV line-segments into $3 \times 3$ matrices [7]. However, in practical LV distribution feeders, neutral grounding resistances can vary from less than $1 \mathrm{ohm}$ to several tens of ohms depending on the type and condition of grounding system [8]-[10]. Further, the unbalance in load and line impedance and the unbalanced allocation of PV resources can produce high neutral current in LV feeders, sometimes even higher than the phase currents [7], [11]. High neutral current can produce significant neutral-to-ground voltage in the presence of neutral grounding resistance. Therefore, the traditional assumption that the neutral-to-ground voltage is zero and merging the neutral line admittances into the phase line admittances in the modeling may not be realistic. High neutral current may produce voltage distortion, and may overload neutral conductor [12]. Further, the neutral-to-ground voltage can act as a common-mode noise in sensitive electronic equipment [13]. Neutral-to-ground voltage beyond manufacturer specified tolerance level [13] may cause mal-operation [14] of sensitive equipment. To avoid such issues, the accurate determination of neutral current and voltage is essential, especially for planning distribution networks with PV integration. Therefore, preserving the 4-wire configuration in the network model would be desirable and development of a power flow approach capable of retaining the original wiring configurations of the MV and LV network segments is necessary.

Extensive research has been performed on 3-phase power flow algorithms for distribution networks with distributed resources [15], however, most of those are based on a 3-wire power flow approach. A forward-backward substitution based 4-wire power flow method is developed in [11] considering the ground-wire impedance. A three-phase 3-wire power flow based on a current-mismatch variant of Newton-Raphson 
algorithm is presented in [16]. Authors in [17] have developed a current-mismatch based 4-wire power flow approach. However, in [17], a combined 3-wire and 4-wire power flow formulation corresponding to $\mathrm{MV}$ and $\mathrm{LV}$ networks is not explicitly presented.

This paper proposes a three-phase power flow approach developed for the analysis of single phase rooftop solar PV impacts on distribution networks by preserving the realistic configurations of MV and LV networks. With this technique, the PV impacts on the host LV network and the upstream MV network can be performed without modifying the model of the original wiring configurations. LV network modeling will be performed based on the original $4 \times 4$ matrix representation of LV line segments, and MV networks will be modeled using $3 \times 3$ matrices. A primitive admittance model of deltawye transformer will be developed based on the technique presented in [18] to satisfy the modeling requirements of the 3-wire (MV) and 4-wire (LV) systems. The integrated system of power flow equations corresponding to the 3wire and 4-wire networks will be solved using the currentmismatch variant of Newton-Raphson algorithm. It is worth mentioning that a network model retaining the original 3wire and 4-wire configurations could be solved using other power flow solution algorithms with minor adjustments to incorporate 3- and 4-wire configuration where the neutral line and ground resistances need to be included. To investigate the impacts of single-phase variable PV generation, a series of power flow calculations will be performed over a 24-hour period. A practical distribution system in Australia will be used to verify the applicability of the proposed approach in real-world distribution networks. Though this paper has concentrated on rooftop PV systems, the problems presented can be experienced with any type of distributed generation resources, such as small residential wind power units or fuel cells, installed in an unbalanced pattern in a distribution feeder. Therefore, the proposed approach can be extended to other distributed generation resources.

This paper is organized into five sections. Section I provides an introduction of the problem of using a three-phase 3-wire power flow approach to analyze 3- and 4-wire configuration often found in MV and LV distribution network. This is particularly important when neutral wire and grounding effects need to be assessed. Network modeling approach with multigrounded LV feeder with rooftop solar PV is discussed in Section II. Formulation of the power flow problem for an integrated 3-wire MV and 4-wire LV network is presented in Section III. The application of the approach has been verified in Section IV using a practical distribution system. Section V concludes the paper by summarizing the works performed and observations made.

\section{System Modeling}

A distribution feeder can be represented as a combination of series elements (overhead and underground line segments, transformers etc.) connected between, and shunt elements (line susceptances, grounding impedance, capacitor / reactor banks, etc.) connected at, different system nodes. Admittance matrix

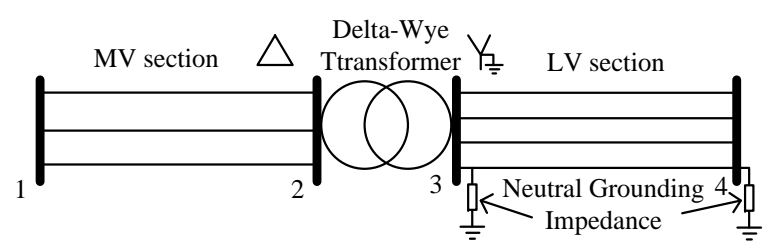

(a) 4-bus, 3-wire and 4-wire test system

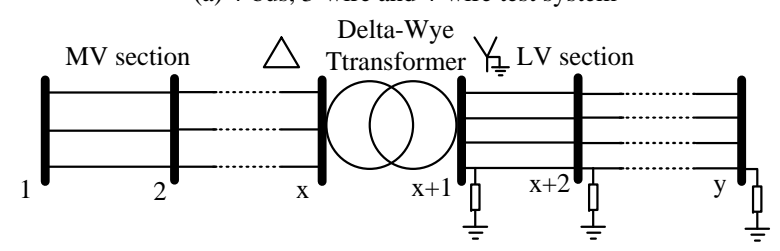

(b) A general extended form of 3-wire and 4-wire test system

Fig. 1. Three Wire and Four Wire Test System

models of the series and shunt components assembled in the form of a total system admittance matrix are used for network analysis. A simple 4-bus distribution test feeder shown in Fig. 1(a), similar to the IEEE 4-bus test system [19] will be used in this paper to formulate the modeling approach of 3-wire and 4-wire systems; a similar approach can be followed for the general extended form of the test system shown in Fig. 1(b). The 4-bus feeder is configured to have a 3-wire MV section from bus 1 to 2, a delta-wye step-down transformer from bus 2 to 3, and a 4-wire LV section from bus 3 to 4. Detailed modeling aspects of the network components are discussed below.

\section{A. Modeling of the MV Lines}

Three wire MV distribution lines can be represented using $3 \times 3$ admittance matrices consisting of the self admittances of three phases $a, b$ and $c$ as the diagonal elements and the mutual coupling admittances among them as off-diagonal elements. Following this model, the MV line admittance of the 4-bus test system, $\mathbf{Y}_{12}^{M V}$, can be written as,

$$
\mathbf{Y}_{12}^{M V}=\mathbf{y}_{i j}^{a b c}=\left[\begin{array}{lll}
y_{i j}^{a a} & y_{i j}^{a b} & y_{i j}^{a c} \\
y_{i j}^{b a} & y_{i j}^{b b} & y_{i j}^{b c} \\
y_{i j}^{c a} & y_{i j}^{c b} & y_{i j}^{c c}
\end{array}\right]
$$

where, the subscripts $i$ and $j$ are used for generic modeling purpose, that represents the buses 1 and 2, respectively, in the network given in Fig. 1(a). It is to be noted that to construct the total system admittance matrix with 3-wire and 4-wire lines, the MV line admittance model needs to be accommodated into the 4-wire line model. Therefore, order of the admittance matrix in (1) is increased to $4 \times 4$ using all zero elements, as given below.

$$
\mathbf{Y}_{12}^{M V}=\left[\begin{array}{cccc}
y_{i j}^{a a} & y_{i j}^{a b} & y_{i j}^{a c} & 0 \\
y_{i j}^{b a} & y_{i j}^{b b} & y_{i j}^{b c} & 0 \\
y_{i j}^{c a} & y_{i j}^{c b} & y_{i j}^{c c} & 0 \\
0 & 0 & 0 & 0
\end{array}\right]
$$

\section{B. Modeling of the LV Lines}

Low voltage distribution feeders are typically constructed with four wire line segments where the fourth wire is the 


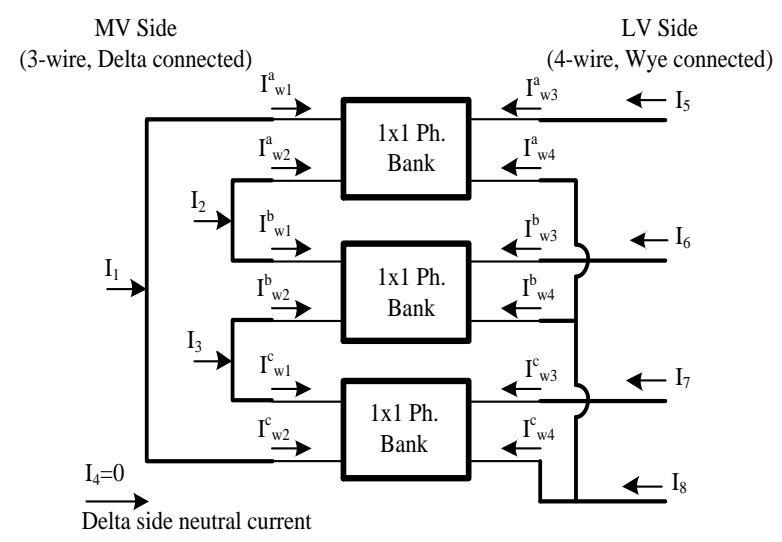

Fig. 2. Terminal connection and currents of delta-wye transformer

neutral wire. Similar to the phase conductors, the neutral conductor has self and mutual impedance components with the other phase wires, and these need to be included in the admittance model. The LV line admittance matrix, $\mathbf{Y}_{34}^{L V}$ of the test system is given below using a $4 \times 4$ matrix model.

$$
\mathbf{Y}_{34}^{L V}=\mathbf{y}_{i j}^{a b c n}=\left[\begin{array}{llll}
y_{i j}^{a a} & y_{i j}^{a b} & y_{i j}^{a c} & y_{i j}^{a n} \\
y_{i j}^{b a} & y_{i j}^{b b} & y_{i j}^{b c} & y_{i j}^{b n} \\
y_{i j}^{c a} & y_{i j}^{c b} & y_{i j}^{c c} & y_{i j}^{c n} \\
y_{i j}^{n a} & y_{i j}^{n b} & y_{i j}^{n c} & y_{i j}^{n n}
\end{array}\right]
$$

Here, $i$ and $j$ are used as subscripts for the generic modeling purpose, similarly as described for (1), which represents buses 3 , and 4 , respectively. The neutral wire is explicitly modeled in this matrix model, as reflected in the $4^{\text {th }}$ row and column.

\section{Modeling of the MV/LV Transformer}

A primitive admittance matrix model of a delta-wye transformer is developed based on the relationship of transformer terminal currents with the internal winding currents, in a similar fashion discussed for wye-delta connection in [18]. The per unit impedance matrix model of a three phase transformer with three phase short circuit impedances, $z_{T}^{a}, z_{T}^{b}$ and $z_{T}^{c}$, can be described in a matrix form as,

$$
\mathbf{Z}_{T}^{a b c}=\left[\begin{array}{ccc}
z_{T}^{a} & 0 & 0 \\
0 & z_{T}^{b} & 0 \\
0 & 0 & z_{T}^{c}
\end{array}\right]
$$

The ground referenced nodal admittance matrix [18], $\mathbf{Y}_{T}^{L}$, can be obtained using the incidence matrix, $B$, relating the short circuit currents with the terminal currents [18], as given below.

$$
\mathbf{Y}_{T}^{L}=B\left(\mathbf{Z}_{T}^{a b c}\right)^{-1} B^{T} \text { and } B=\left[\begin{array}{ccc}
1 & 0 & 0 \\
-1 & 0 & 0 \\
0 & 1 & 0 \\
0 & -1 & 0 \\
0 & 0 & 1 \\
0 & 0 & -1
\end{array}\right]
$$

The winding admittance matrix $\mathbf{Y}_{T}^{W}$ can be obtained from $\mathbf{Y}_{T}^{L}$ by using the following equation,

$$
\mathbf{Y}_{T}^{W}=N \mathbf{Y}_{T}^{L} N^{T}
$$

$$
\begin{gathered}
\text { where, } N=\left[\begin{array}{ccc}
N^{a} & \mathbf{0} & \mathbf{0} \\
\mathbf{0} & N^{b} & \mathbf{0} \\
\mathbf{0} & \mathbf{0} & N^{c}
\end{array}\right] \\
\text { and } N^{a}=N^{b}=N^{c}=\left[\begin{array}{cc}
1 & 0 \\
-1 & 0 \\
0 & 1 \\
0 & -1
\end{array}\right]
\end{gathered}
$$

In (7), $\mathbf{0}$ denotes a $4 \times 2$ matrix of zero elements, and in (8) 1 corresponds to nominal per unit turns ratio. The terminal currents in the delta-wye transformer shown in Fig. 2 can be related to the winding currents by,

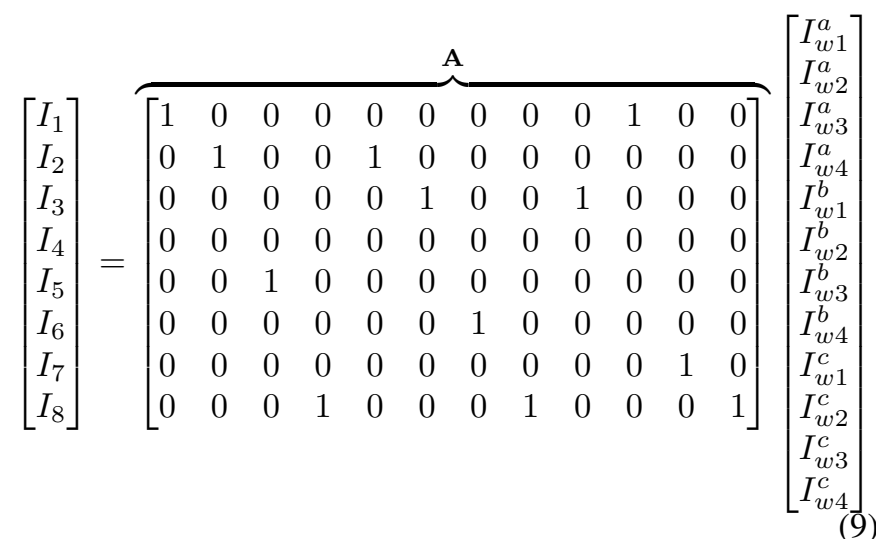

It is to be noted that the $4^{\text {th }}$ row of $\mathbf{A}$, corresponding to the neutral current of delta side, is a vector of all zero elements as there is no neutral current for the delta side. This row is inserted for making the 3-wire side of the admittance matrix compatible with the 4-wire system calculation, as discussed earlier in the MV and LV line modeling. The primitive admittance matrix of the transformer, $\mathbf{Y}_{T}^{\text {prim }}$, is found as,

$$
\mathbf{Y}_{T}^{p r i m}=\mathbf{A} \mathbf{Y}_{T}^{W} \mathbf{A}^{T}=\left[\begin{array}{ll}
\mathbf{Y}_{T}^{P P} & \mathbf{Y}_{T}^{P S} \\
\mathbf{Y}_{T}^{S P} & \mathbf{Y}_{T}^{S S}
\end{array}\right]
$$

where,
$\mathbf{Y}_{T}^{P}$,
$\mathbf{Y}_{T}^{S}$

is the primary (delta) side self admittance matrix is the secondary (wye) side self admittance matrix

$\mathbf{Y}_{T}^{P S}, \mathbf{Y}_{T}^{S P}$ are the mutual admittance matrices between primary and secondary sides

To develop the per-unit model, $\mathbf{Y}_{T}^{P S}$ and $\mathbf{Y}_{T}^{S P}$, each has to be divided by $\sqrt{3}$ and $\mathbf{Y}_{T}^{P P}$ has to be divided by 3, as described in [20].

\section{Admittance Matrix of the Integrated System}

The total system admittance matrix, $\mathbf{Y}$, for a $k$-bus three phase power system can be constructed as a $4 k \times 4 k$ matrix to accommodate both 3-wire and 4-wire admittance matrices. The diagonal and off-diagonal elements of $\mathbf{Y}$ are obtained by,

$$
\mathbf{Y}_{i i}=\sum_{i=1}^{k} \mathbf{y}_{i j}^{a b c n} \text { and, } \mathbf{Y}_{i j}=-\mathbf{y}_{i j}^{a b c n}
$$

where, $i$ and $j=1,2, \cdots, k$.

Self admittance matrices of the transformer primary and secondary are added with the self admittance matrices of 
the primary bus and the secondary bus, respectively, and the mutual admittances are added with the mutual admittances between primary and secondary buses. Following this method, the system admittance matrix for the 4-bus test system with integrated MV and LV networks can be written as,

$$
\mathbf{Y}=\left[\begin{array}{cccc}
\mathbf{Y}_{12}^{M V} & -\mathbf{Y}_{12}^{M V} & 0 & 0 \\
-\mathbf{Y}_{12}^{M V} & \mathbf{Y}_{12}^{M V}+\mathbf{Y}_{T}^{P P} & -\mathbf{Y}_{T}^{P S} & 0 \\
0 & -\mathbf{Y}_{T}^{S P} & \mathbf{Y}_{34}^{L V}+\mathbf{Y}_{T}^{S S} & -\mathbf{Y}_{34}^{L V} \\
0 & 0 & -\mathbf{Y}_{34}^{L V} & \mathbf{Y}_{34}^{L V}
\end{array}\right]
$$

Buses with grounded neutral, such as bus 3 and 4 in Fig. 1(a), are modeled by adding the neutral grounding admittances with the self admittances of the neutral wire at the respective buses. This is given in the equation below for an arbitrary bus $i$, where the neutral grounding admittance is $y_{i i}^{N-G}$.

$$
\mathbf{Y}_{i i}=\mathbf{Y}_{i i}+\left[\begin{array}{cccc}
0 & 0 & 0 & 0 \\
0 & 0 & 0 & 0 \\
0 & 0 & 0 & 0 \\
0 & 0 & 0 & y_{i i}^{N-G}
\end{array}\right]
$$

\section{E. Modeling of Loads}

Realistic modeling of distribution network loads is a complex task involving comprehensive study of the types, ratings and consumption trends of electrical appliances. Lighting, cooking, cooling, heating, use of computer, telecommunication and entertainment systems are some of the common types of electricity usage in residential households and all of these applications vary throughout the day based on consumer behavior. Practical modeling of distribution feeder loads, especially at LV level, would therefore require modeling of load variations of each of the individual end-use applications. Total load profile of the households then could be performed by aggregation of the individual load profiles. ZIP representation of typical residential loads [21], [22] will be used for modeling the loads in this paper. A ZIP load model includes proportions of constant power, constant current, and constant impedance loads as given below.

$$
\begin{aligned}
& P^{\mathrm{ZIP}}=P_{0}\left(p^{\mathrm{P}}+\left|\frac{V_{P h-N}}{V_{0}}\right| p^{\mathrm{I}}+\left|\frac{V_{P h-N}}{V_{0}}\right|^{2} p^{\mathrm{Z}}\right) \\
& Q^{\mathrm{ZIP}}=Q_{0}\left(q^{\mathrm{P}}+\left|\frac{V_{P h-N}}{V_{0}}\right| q^{\mathrm{I}}+\left|\frac{V_{P h-N}}{V_{0}}\right|^{2} q^{\mathrm{Z}}\right)
\end{aligned}
$$

where, $p^{\mathrm{P}}+p^{\mathrm{I}}+p^{\mathrm{Z}}=1$ and $q^{\mathrm{P}}+q^{\mathrm{I}}+q^{\mathrm{Z}}=1$

Here, $P_{0}$ and $Q_{0}$ are the nominal real and reactive power at nominal voltage $V_{0}$; and $p^{\mathrm{P}}, p^{\mathrm{I}}, p^{\mathrm{Z}}$, and $q^{\mathrm{P}}, q^{\mathrm{I}}, q^{\mathrm{Z}}$ are respectively the constant power, constant current and constant impedance proportions of active and reactive load and termed as ZIP parameters, and $V_{P h-N}$ is the phase-to-neutral voltage. To obtain the aggregate load model for the household, aggregate ZIP parameters for active and reactive components of loads, $\mathrm{ZIP}_{a g g}^{P}$ and $\mathrm{ZIP}_{a g g}^{Q}$, where $Z I P^{P} \in\left\{p^{\mathrm{P}}, p^{\mathrm{I}}, p^{\mathrm{Z}}\right\}$ and $\operatorname{ZIP}^{Q} \in\left\{q^{\mathrm{P}}, q^{\mathrm{I}}, q^{\mathrm{Z}}\right\}$, will be determined using weighted average of individual ZIP parameters, as given below.

$$
\mathrm{ZIP}_{\text {agg }}^{P}=\sum_{i=1}^{n}\left(\frac{P_{i}}{P_{\text {total }}}\right) \mathrm{ZIP}_{i}^{P}
$$

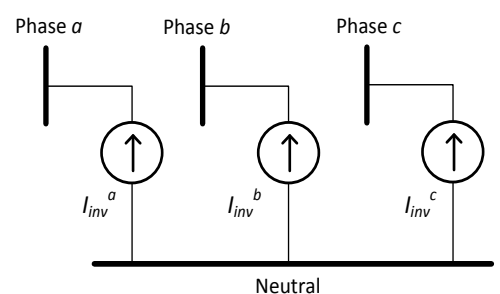

Fig. 3. Current injection model of solar PV

$$
\mathrm{ZIP}_{\text {agg }}^{Q}=\sum_{i=1}^{n}\left(\frac{Q_{i}}{Q_{\text {total }}}\right) \mathrm{ZIP}_{i}^{Q}
$$

Here, $i=1,2, \ldots n$, for $n$ number of loads corresponding to different household activities.

\section{F. Modeling of Rooftop Solar PV}

Solar PV systems installed in LV feeders typically contain rooftop PV modules and associated single phase inverter modules. The inverter output is connected between the phase and neutral conductor, as shown in Fig. 3 for three single phase PV sources connected at phases $a, b$ ands $c$. Current injection of PV inverter, $I_{i n v}$, depends on the complex output power of the inverter, $P_{i n v}+j \sqrt{S_{i n v}^{2}-P_{i n v}^{2}}$, where, $P_{i n v}$ is the inverter real power, $S_{i n v}$ is the PV inverter apparent power capacity, and $V_{P h-N}$ is the phase-to-neutral voltage.

$$
I_{i n v}=\left(\frac{P_{i n v}+j \sqrt{S_{i n v}^{2}-P_{i n v}^{2}}}{V_{P h-N}}\right)^{*}
$$

Here, * represents complex conjugate. It is to be noted that $S_{i n v}$ is not directly related to the DC power coming out from the PV modules. The $P_{i n v}$ is the AC power generated by the inverter for a given value of DC power generated by the PV modules, $P_{D C}$, taking into account efficiency of the inverter, $\eta_{\text {inv }}$, mismatch among multiple PV modules, $\eta_{m}$, and dirt effects, $\eta_{d}$, as given below [23].

$$
P_{i n v}=\eta_{i n v} \times \eta_{m} \times \eta_{d} \times P_{D C}
$$

The inverter controls the amount of reactive power output that can be generated from the inverter, and is given by $\sqrt{S_{i n v}^{2}-P_{i n v}^{2}}$. For a larger reactive power output required from the inverter, $S_{i n v}$ should be made larger, for the same $P_{\text {inv. }} P_{D C}$ can be obtained from the $I-V$ characteristic of the solar PV module as given by the following equation,

$$
P_{D C}=\max \left(V_{D C} \times I_{D C}\right)
$$

where, $V_{D C}$ is the PV module voltage, $I_{D C}$ is the PV module current and $\max (\cdot)$ implements the Maximum Power Point Tracking (MPPT) function. The function $\max ($.$) given$ in (18), is a function used to select the maximum value from a series of $V_{D C}$ times $I_{D C}$ values to obtain the maximum power point of the $P-V$ characteristic, as shown in Fig. 4. PV module voltage $V_{D C}$, depends on ambient conditions and PV module electrical parameters, whereas the PV module current $I_{D C}$ depends on the ambient conditions, the module 
(a)
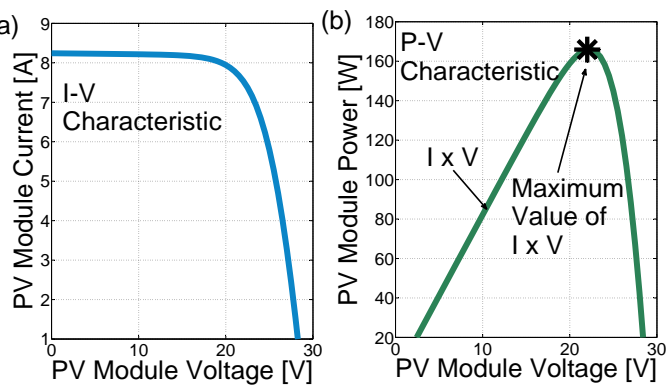

Fig. 4. Implementation of MPPT function; (a) $I-V$ characteristic (b) $P-V$ characteristic

voltage, and the PV current itself [24]. Mathematically this can be described as,

$$
\begin{aligned}
& V_{D C}=\phi(t, \mathbf{p}) \\
& I_{D C}=\psi\left(t, G, \mathbf{p}, V_{D C}, I_{D C}\right)
\end{aligned}
$$

where,

$t \quad$ is the ambient temperature

$G \quad$ is the ambient sun irradiance level

p is a vector of PV module electrical parameters, such as module resistances, short-circuit current, voltage-temperature and current-temperature coefficients etc.

$\phi \quad$ is a function to determine the PV module voltage from ambient conditions and PV parameters

$\psi \quad$ is a function to determine the PV module current from ambient conditions, PV parameters, module voltage and module current

An observation of (20) indicates that it is a transcendental expression and an analytical form of solution is not available [24]. Therefore, numerical technique is applied to solve (20) to find the I-V characteristic of PV modules at any given ambient conditions. The I-V characteristic is then used to find out the current injection from PV inverters into the network.

\section{Proposed 3-Phase Power Flow for Integrated 3-Wire AND 4-Wire SySTEMS WITH SOLAR PV}

For a converged power flow problem, the mismatch between specified current and calculated current should be nearly zero. Such mismatch, denoted by $\Delta \mathbf{I}_{m}$ at an arbitrary bus $m$ of a three phase feeder can be expressed as,

$$
\Delta \mathbf{I}_{m}=\left(\mathbf{I}_{m}\right)^{\text {spec }}-\left(\mathbf{I}_{m}\right)^{\text {calc }} \approx 0
$$

Here, $\left(\mathbf{I}_{m}\right)^{\text {spec }}$ is the vector of specified currents and $\left(\mathbf{I}_{m}\right)^{\text {calc }}$ is the vector of calculated currents at the bus $m$. If the three phase bus $m$ is of 3-wire configuration, then the length of the mismatch vector and the current vectors is 3 , and, for a 4-wire configuration the length is 4 , where the $4^{\text {th }}$ element corresponds to the neutral current. Specified currents at bus $m$ can be obtained from current contributions from ZIP load and solar PV as given in (22) based on the current injection model shown in Fig. 5.

$$
\left(\mathbf{I}_{m}\right)^{\text {spec }}=-\mathbf{I}_{m}^{\mathrm{ZIP}}+\mathbf{I}_{m}^{\mathrm{PV}}
$$

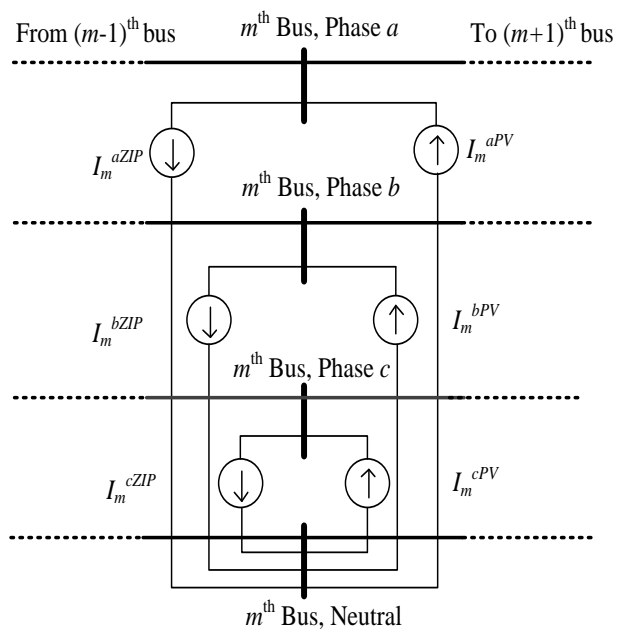

Fig. 5. Current injection model of load and PV for power flow formulation

$$
\begin{aligned}
& \text { where, } \mathbf{I}_{m}^{\mathrm{ZIP}}=\left(\frac{\mathbf{P}_{m}^{\mathrm{ZIP}}+j \mathbf{Q}_{m}^{\mathrm{ZIP}}}{\mathbf{V}_{m}}\right)^{*} \\
& \text { and, } \mathbf{I}_{m}^{\mathrm{PV}}=\left(\frac{\mathbf{P}_{m}^{\mathrm{PV}}+j \mathbf{Q}_{m}^{\mathrm{PV}}}{\mathbf{V}_{m}}\right)^{*}
\end{aligned}
$$

where,

$$
\begin{array}{ll}
\mathbf{I}_{m}^{\mathrm{PV}} & \begin{array}{l}
\text { is the vector of phase currents con- } \\
\text { tributed by solar PV at phases } a, b, c
\end{array} \\
\mathbf{P}_{m}^{\mathrm{ZIP}}+j \mathbf{Q}_{m}^{\mathrm{ZIP}} & \begin{array}{l}
\text { is the vector of complex powers corre- } \\
\text { sponding to loads at phases } a, b, c
\end{array} \\
\mathbf{P}_{m}^{\mathrm{PV}}+j \mathbf{Q}_{m}^{\mathrm{PV}} & \begin{array}{l}
\text { is the vector of complex powers corre- } \\
\text { sponding to solar PV inverters at phases }
\end{array} \\
& \begin{array}{l}
a, b, c \\
\text { is the vector of complex voltages at } \\
\text { phases } a, b, c
\end{array}
\end{array}
$$

The minus sign in (22) with $\mathbf{I}_{m}^{\mathrm{ZIP}}$ represents the difference in the direction of current injections from the ZIP load and solar PV. In (24), $\mathbf{Q}_{m}^{\mathrm{PV}}$ will be zero if unity power factor operation of $\mathrm{PV}$ is considered. It is to be noted that for 3-wire networks, $\mathbf{V}_{m}$ comprises of the phase voltages, whereas, for 4-wire networks it contains the phase-to-neutral voltages. The specified current through the neutral wire, $\left(I_{m}^{N}\right)^{\text {spec }}$ can be found by adding the specified phase currents $I_{m}^{a}, I_{m}^{b}$ and $I_{m}^{c}$, obtained from the vector of specified phase currents, $\left(\mathbf{I}_{m}\right)^{\text {spec }}$.

$$
\left(I_{m}^{N}\right)^{s p e c}=-\left(I_{m}^{a}+I_{m}^{b}+I_{m}^{c}\right)
$$

The calculated currents can be obtained using the total system admittance matrix and the vector of system voltages using the following classical network current equation.

$$
\left(\mathbf{I}_{m}\right)^{\text {calc }}=\sum_{n=1}^{k} \mathbf{Y}_{m n} \mathbf{V}_{n}
$$

Here, $\mathbf{Y}_{m n}$ is the admittance matrix from bus $m$ to $n$, where, $n=1,2, \ldots, k$, and $k$ is the total number of system buses. Mismatch in the specified and calculated current injection components in phase and neutral nodes of a three phase 
feeder can be expressed in terms of their real and imaginary components using the following equations.

$$
\begin{aligned}
\Delta \mathbf{I}_{m_{R e}} & =\frac{\mathbf{P}_{m} \mathbf{V}_{m_{R e}}+\mathbf{Q}_{m} \mathbf{V}_{m_{I m}}}{\left(\left|\mathbf{V}_{m_{R e}}+j \mathbf{V}_{m_{I m}}\right|\right)^{2}} \\
& -\sum_{n=1}^{k}\left(\mathbf{G}_{m n} \mathbf{V}_{n_{R e}}-\mathbf{B}_{m n} \mathbf{V}_{n_{I m}}\right) \\
\Delta \mathbf{I}_{m_{I m}} & =\frac{\mathbf{P}_{m} \mathbf{V}_{m_{I m}}-\mathbf{Q}_{m} \mathbf{V}_{m_{R e}}}{\left(\left|\mathbf{V}_{m_{R e}}+j \mathbf{V}_{m_{I m}}\right|\right)^{2}} \\
& -\sum_{n=1}^{k}\left(\mathbf{G}_{m n} \mathbf{V}_{n_{I m}}+\mathbf{B}_{m n} \mathbf{V}_{n_{R e}}\right) \\
\Delta I_{m_{R e}}^{N} & =-\left(I_{m_{R e}}^{a}+I_{m_{R e}}^{b}+I_{m_{R e}}^{c}\right) \\
& -\sum_{n=1}^{k}\left(\mathbf{G}_{m n}^{N} \mathbf{V}_{n_{R e}}-\mathbf{B}_{m n}^{N} \mathbf{V}_{n_{I m}}\right) \\
\Delta I_{m_{I m}}^{N} & =-\left(I_{m_{I m}}^{a}+I_{m_{I m}}^{b}+I_{m_{I m}}^{c}\right) \\
& -\sum_{n=1}^{k}\left(\mathbf{G}_{m n}^{N} \mathbf{V}_{n_{I m}}+\mathbf{B}_{m n}^{N} \mathbf{V}_{n_{R e}}\right)
\end{aligned}
$$

The subscripts $R e$ and ${ }_{I m}$ in (27)-(30) stand for Real and Imaginary, respectively. In (27) and (28), $\mathbf{G}_{m n}$ and $\mathbf{B}_{m n}$ are $3 \times 4$ matrices consisting of the corresponding real and imaginary parts of the admittance matrix from bus $m$ to $n$, with respect to the phase conductors. In (29) and (30), $\mathbf{G}_{m n}^{N}$ and $\mathbf{B}_{m n}^{N}$ are $1 \times 4$ vectors consisting of the respective real and imaginary parts of the admittance matrix from bus $m$ to $n$ corresponding to the neutral conductor. The system of non-linear equations (27)-(30) can be solved using NewtonRaphson (N-R) iterative algorithm that needs to relate the incremental changes in voltages with incremental changes in currents in terms of real and imaginary components, as given below.

$$
\left[\begin{array}{c}
\Delta \mathbf{V}_{m_{R e}} \\
\Delta V_{m_{R e}^{N}} \\
\Delta \mathbf{V}_{m_{I m}} \\
\Delta V_{m_{I m}^{N}}^{N}
\end{array}\right]=i n v(\mathbf{J}) \times\left[\begin{array}{c}
\Delta \mathbf{I}_{m_{I m}} \\
\Delta I_{m_{I m}^{N}} \\
\Delta \mathbf{I}_{m_{R e}} \\
\Delta I_{m_{R e}^{N}}^{N}
\end{array}\right]
$$

The incremental voltage vector in (31) refers to the updates in voltages to be used in the power flow iterations of N-R algorithm, and the incremental current vector refers to the current mismatches. The $1^{\text {st }}$ and $3^{\text {rd }}$ row of voltage vector contains the real and imaginary components of phase voltage updates, respectively, and the $2^{\text {nd }}$ and $4^{\text {th }}$ row contains the updates of neutral voltage; $\mathbf{J}$ is the Jacobian matrix. For 3-wire segments of distribution networks, the neutral components are not present and therefore, the $2^{\text {nd }}$ and $4^{\text {th }}$ rows are not included in the N-R equations. Following this, the N-R equation for the 4-bus test system under consideration can written as,

$$
\left[\begin{array}{c}
\Delta V_{2}^{a b c} \\
\Delta V_{3}^{a b c n} \\
\Delta V_{4}^{a b c n}
\end{array}\right]=i n v\left(\left[\begin{array}{lll}
J_{22}^{a b c} & J_{23}^{a b c n} & J_{24}^{a b c n} \\
J_{32}^{a b c n} & J_{33}^{a b c n} & J_{34}^{a b c n} \\
J_{42}^{a b c n} & J_{43}^{a b c n} & J_{44}^{a b c n}
\end{array}\right]\right) \times\left[\begin{array}{c}
\Delta I_{2}^{a b c} \\
\Delta I_{3}^{a b c n} \\
\Delta I_{4}^{a b c n}
\end{array}\right]
$$

where,
$\Delta V_{2}^{a b c}$ is the 6-element incremental voltage vector consisting of $\left[\Delta V_{2 R e}^{a b c} \quad \Delta V_{2_{I m}}^{a b c}\right]^{T}$, corresponding to the 3-wire bus, Bus-2. $T$ represents transpose.

$\Delta V_{3}^{a b c n}$ is the 8-element incremental voltage vector consisting of $\left[\Delta V_{3_{R e}}^{a b c n} \quad \Delta V_{3_{I m}}^{a b c n}\right]^{T}$, corresponding to the 4-wire bus, Bus-3. The similar notations hold for the other 4-wire bus, bus 4 .

$\Delta I_{2}^{a b c} \quad$ is the 6-element current mismatch vector consisting of $\left[\Delta I_{2_{I m}}^{a b c} \quad \Delta I_{2_{R e}}^{a b c}\right]^{T}$, corresponding to the 3-wire bus, Bus-2.

$\Delta I_{3}^{a b c n} \quad$ is the 8-element current mismatch vector consisting of $\left[\Delta I_{3_{I m}}^{a b c n} \quad \Delta I_{3 R e}^{a b c n}\right]^{T}$, corresponding to the 4-wire bus, Bus-3. The similar notations hold for the other 4-wire bus, bus 4 .

The first element in the Jacobian matrix, $J_{22}^{a b c}$, is a $6 \times 6$ matrix block corresponding to the 3 wire section of the system, while $J_{33}^{a b c n}, J_{34}^{a b c n}, J_{43}^{a b c n}$ and $J_{44}^{a b c n}$ are $8 \times 8$ matrix blocks corresponding to the four wire section. The remaining, $J_{23}^{a b c n}, J_{24}^{a b c n}$ and $J_{32}^{a b c n}, J_{42}^{a b c n}$ are, respectively, 6x8 and 8x6 matrices, corresponding to the delta-wye transformer between the three wire and four wire network segments. It is to be noted that Bus 1, being the slack bus, has been excluded from (32). Following a similar type of notations, the N-R equation of the general form of 3-wire and 4-wire feeder in fig. 1(b) containing $x$ number of 3-wire buses and $(y-x)$ number of 4-wire buses can be developed as given below.

$$
\left[\begin{array}{c}
\Delta V_{2}^{a b c} \\
\vdots \\
\Delta V_{x}^{a b c} \\
\Delta V_{x+1}^{a b c n} \\
\vdots \\
\Delta V_{y}^{a b c n}
\end{array}\right]=i n v(\mathbf{J}) \times\left[\begin{array}{c}
\Delta I_{2}^{a b c} \\
\vdots \\
\Delta I_{x}^{a b c} \\
\Delta I_{x+1}^{a b c n} \\
\vdots \\
\Delta I_{y}^{a b c n}
\end{array}\right]
$$

where,

$$
\mathbf{J}=\left[\begin{array}{cc}
J_{22}^{a b c} \cdots J_{2 x}^{a b c} & J_{2(x+1)}^{a b c n} \cdots J_{2 y}^{a b c n} \\
\vdots \ddots \vdots & \vdots \ddots \vdots \\
J_{x 2}^{a b c} \cdots J_{x x}^{a b c} & J_{x(x+1)}^{a b c n} \cdots J_{x y}^{a b c n} \\
J_{(x+1) 2}^{a b c n} \cdots J_{(x+1) x}^{a b c n} & J_{(x+1)(x+1)}^{a b c n} \cdots J_{(x+1) y}^{a b c n} \\
\vdots \ddots \vdots & \vdots \ddots \vdots \\
J_{y 2}^{a b c n} \cdots J_{y x}^{a b c n} & J_{y(x+1)}^{a b c n} \cdots J_{y y}^{a b c n}
\end{array}\right]
$$

Methods of calculating Jacobian elements described in [16], [17] will be deployed in this paper. Updates of real and imaginary components of voltages are found using (33) in each iteration of the N-R algorithm that continues until the current mismatch in (21) is satisfied with an acceptable tolerance. This power flow approach using N-R algorithm works for both mesh and radial networks. 


\section{Application of the Proposed 3-Phase Power FLOW APPROACH TO ASSESS PV IMPACTS}

\section{A. The IEEE 4-bus Test Case}

The IEEE 4-bus system [19] is a standard distribution test feeder to verify power flow programs with different transformer connections. The unbalanced loading with delta-wye step-down transformer connection is used in this paper. The $12.47 \mathrm{kV}$ line segment is configured as 3-wire and the $4.16 \mathrm{kV}$ segment is configured as 4-wire. Power flow results for the 3 wire and 4-wire segments of the test system obtained using the proposed approach are presented in Table I. Neutral voltages in the order of $10^{-3} \mathrm{~V}$ are obtained for a solidly grounded neutral $\left(10^{-4} \mathrm{ohm}\right)$ and are assumed as zero, as shown in Table I for the 4-wire buses, 3 and 4. On the other hand, even a low grounding resistance of $0.3 \mathrm{ohm}$, which is within acceptable limit [7], produced a $74 \mathrm{~V}$ of neutral voltages at bus 3 and 4 in the presence of high unbalance in loads. The neutral voltages presented in Table I cannot be directly obtained as a power flow result using a traditional 3-wire power flow approach. This neutral voltage calculation capability would be useful for distribution feeders with imperfect neutral grounding and containing unbalanced integration of solar PV.

TABLE I

IEEE-4 Bus Test Results with DifFERENT GRounding Options

\begin{tabular}{||l||c||r||r||r||}
\hline Grounding Type & Ph & Bus 2 (V) & Bus 3 (V) & Bus 4 (V) \\
\hline \hline \multirow{4}{*}{ Solidly Grounded } & A & 12350 & 2293 & 2163 \\
& B & 12360 & 2263 & 1939 \\
& C & 12285 & 2206 & 1837 \\
& N & N/A & $\approx 0$ & $\approx 0$ \\
\hline \multirow{4}{*}{ Resistance Grounded } & A & 12350 & 2325 & 2161 \\
& B & 12363 & 2316 & 2012 \\
& C & 12271 & 2105 & 1719 \\
& N & N/A & 74 & 74 \\
\hline
\end{tabular}

\section{B. A Practical Test Network}

The proposed power flow approach is tested on a real distribution feeder in New South Wales (NSW), Australia. It is important to note that the introduction of non-metallic underground water reticulation system in Australia is contributing to increased grounding impedance in the LV feeders, as many of the service drops are bonded with previously metallic water piping system for neutral grounding purpose. Unbalanced PV allocation at a high penetration level will produce increased neutral current and hence will increase the neutral voltage in the presence of elevated neutral impedance. Therefore, the proposed power flow approach could be useful for PV impact analysis on such a network with high neutral grounding impedances. The test feeder is an $80 \mathrm{~km}$ long $11 \mathrm{kV}$ rural feeder with 3 series voltage regulators, as shown in Fig. 5. The $0.4 \mathrm{kV} \mathrm{LV}$ feeders are connected at different buses along the MV feeders through $11 / 0.4 \mathrm{kV}$ delta-wye transformers. Residential loads in the LV feeders are distributed in an unbalanced pattern among the phases. Load data measured on a practical Australian feeder are used for simulation. PV resources are integrated in a clustered form, i.e., numerous PV units are installed in a small geographical area. Transformer

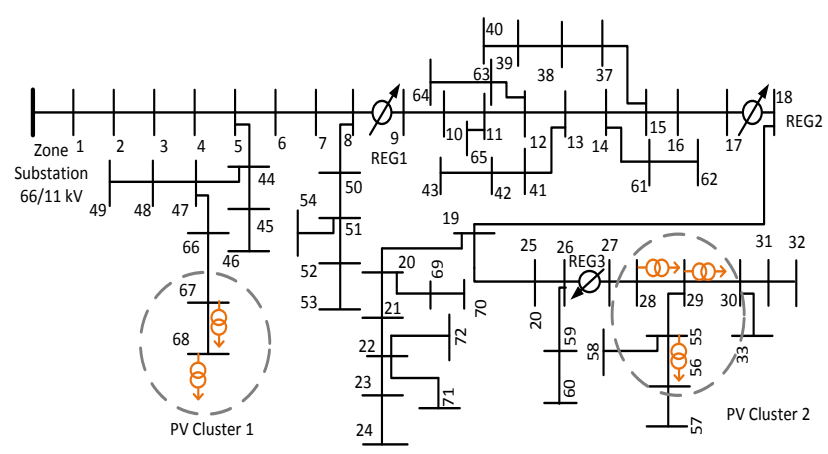

Fig. 6. A practical distribution feeder in NSW

symbols are used to identify the feeders that form the PV clusters. Size of PV units at different residential households ranges from 2 to $5 \mathrm{~kW}$. PV outputs are generated using the April irradiance data given in [23] and applied to obtain the I-V characteristic of Kyocera KC200GT [25] PV module. Numerical values of neutral grounding resistances in PV cluster 1 have been set much higher than the ones in PV cluster 2 for analyzing the effects of high neutral grounding resistance. Analysis is performed using both idealistic and realistic values of neutral to ground resistance in PV cluster 2. IEEE Std. 182-1991 [8]suggests that neutrals of LV systems are typically grounded solidly. For a solidly grounded neutral, phase-to-ground fault current can be equal to $100 \%$ or greater than the three-phase fault current [8]. Based on this criterion, and using the MV/LV substation and LV feeder data of the test system under study, an idealistic value of the neutral to ground resistance for solidly grounded neutral was determined as $0.05 \mathrm{ohm}$. A realistic value of $0.5 \mathrm{ohm}$ is selected according to the recommended value of neutral grounding resistance in a Combined Multiple Earthed Neutral (CMEN) system for Australian distribution utilities [9], [10]. To consider a 10times increase in neutral grounding resistance due to bonding with non-metallic water piping system as discussed in [26], neutral grounding resistance of $5 \mathrm{ohm}$ is used in PV cluster 1.

\section{PV Impacts on LV Networks}

Solar PV resources generate at their peak capacity during midday depending on the ambient sun insolation and temperature. Household load demand is comparatively lower during this time. Power generation from PV resources, therefore, may exceed the load level at the PV connection point at this time, and voltage rise may be observed. Such a scenario is shown in Fig. 7 at one of the LV feeder ends selected from both of the PV clusters in the network. The upper plot in Fig. 7(a) shows voltage from PV cluster 1, which shows significant rise in voltage at midday, and phase $a$ voltage exceeds the upper limit. The lower plot in Fig. 7(b) shows the voltage rise in PV cluster 2 , which is maintained within the limit by the action of voltage regulator REG3 (in Fig. 6).

Due to unbalanced allocation of PV units, voltage rise is different at different phases of the feeder. This unbalance in power injection produces significant increase of neutral current in the LV feeder at midday as shown in Fig. 8. The upper plot 
(a) Voltage Rise in PV Cluster 1

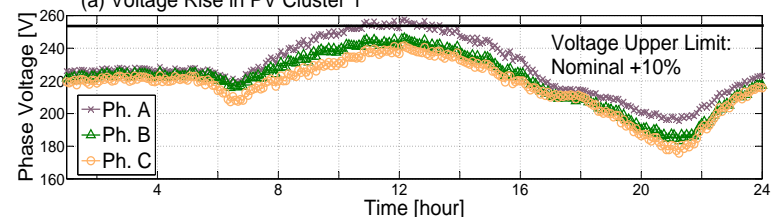

(b) Voltage Rise in PV Cluster 2

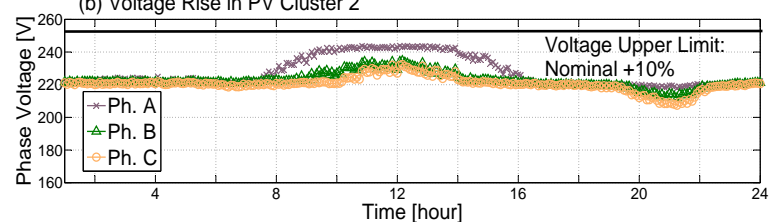

Fig. 7. Daily voltage profile at LV feeder end (a) PV cluster 1 (b) PV cluster

in Fig. 8(a) shows the phase and neutral currents at an LV substation bus in PV cluster 1, and the lower plot in Fig. 8(b) shows the same for PV cluster 2. It is observed that in PV cluster 1 , the neutral current exceeds phase $b$ and $c$ currents, and nearly reaching the phase $a$ current at midday. The situation is less severe in PV cluster 2 with much lower grounding resistance. The high neutral current produced by unbalanced PV injection increases the neutral voltage as shown in Fig. 9; the upper plot in Fig. 9(a) shows neutral voltage in PV cluster 1 that remains nearly at $1 \mathrm{~V}$ during high PV generation period. This is already higher than the commonmode noise limit of $0.5 \mathrm{~V}$ [13] for sensitive equipment. With the idealistic value of neutral resistance of $0.05 \mathrm{ohm}$ in PV cluster 2 , the neutral voltage in cluster 2 does not exceed $0.15 \mathrm{~V}$, as shown in Fig. 9(b). However, using the realistic value of $0.5 \mathrm{ohm}$ in PV cluster 2, the neutral voltage in cluster 2 slightly exceeds the common-mode voltage limit of $0.5 \mathrm{~V}$. Such neutral to ground voltages may exceed the tolerance level with further increase in unbalanced PV allocation which would impose potential safety hazards and may also cause damage of sensitive equipments [13], [14].

A case study is conducted for a scenario where the total PV generation in the feeder is higher than the feeder demand and results are presented in Fig. 10. In this case, surplus power from PV resources is injected into the upstream MV network. This is shown in the upper plot of Fig. 10(a) with a reverse power flow at one of the LV substation buses in a PV cluster. This suggests that a voltage rise is created in the MV feeder at the immediate upstream of the PV clusters due to the reverse power flow. The reactive power flow at the substation bus, however, remains nearly unchanged as shown in the lower plot of Fig. 10(b), because the PV inverters are modeled to operate at unity power factor.

It is to be noted, if the neutral grounding resistances are approximated to zero, then the power flow results obtained by including the neutral wire would not be different than the results obtained using a 3-wire representation of a 4-wire LV feeder. The grounding resistances need to be non-zero to observe the effect of including neutral wire in network model.

\section{PV Impacts Propagated to MV Networks}

$\mathrm{PV}$ impact propagation into the MV network is presented in this paper using MV level voltage profiles. Fig. 11 shows the

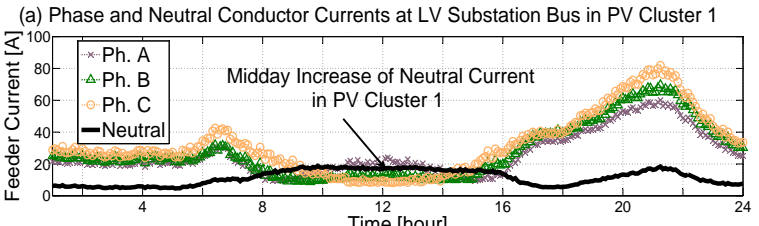

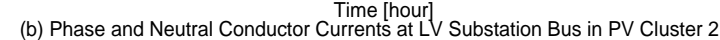

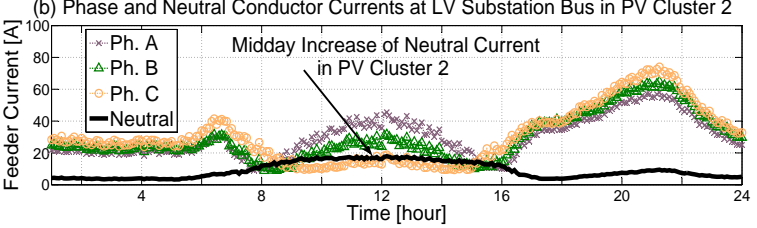

Fig. 8. Daily variation of neutral current (a) PV cluster 1 (b) PV cluster 2

(a) Neutral Voltage in PV Cluster 1

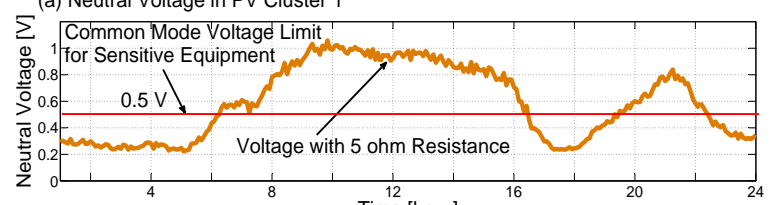

(b) Neutral Voltage in PV Cluster 2 Time [hour]

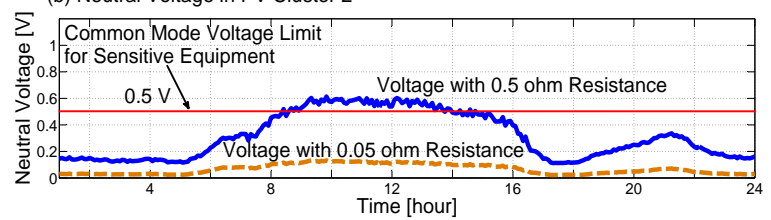

Fig. 9. Daily variation of neutral voltage (a) PV cluster 1 (b) PV cluster 2
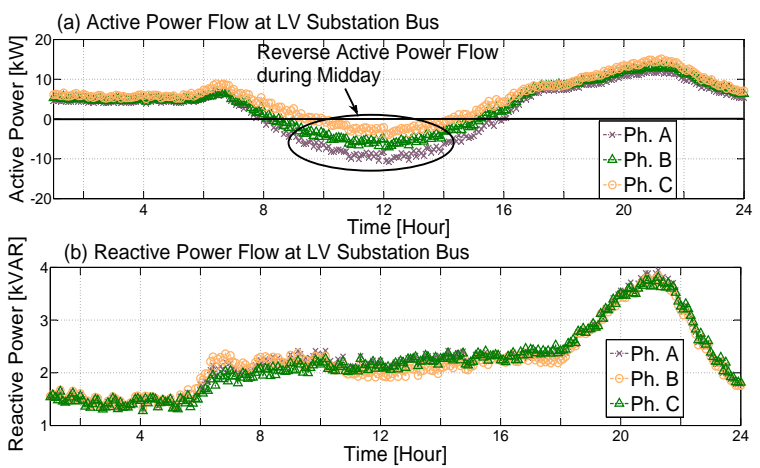

Fig. 10. Daily variation of active and reactive power flow at substation node (a) active power (b) reactive power

voltage profiles of the MV feeder along the feeder length that contains the two PV clusters. This voltage profile corresponds to the time of peak PV generation in the cluster. Voltage rise is observed along one of the spurs of the MV feeder (through the buses 47, 66, 67 and 68 in Fig. 5) due to the absence of voltage regulator at the immediate upstream. On the other hand, voltage rise produced by the PV cluster 2 is bucked down by REG3. This effect is also observed in Fig. 12 that shows the tap operations of the REG3; Fig. 12(a), (b) and (c) shows the tappings of phase $a, b$, and $c$, respectively. The positive tap numbers indicate voltage bucking operation, whereas, the negative tap numbers mean voltage boosting. It is observed that at midday, from about $11 \mathrm{AM}$ to $1 \mathrm{PM}$, the tapping of phase $a$ is positive that indicates the action of REG3 to reduce the voltage rise caused by the PV cluster 


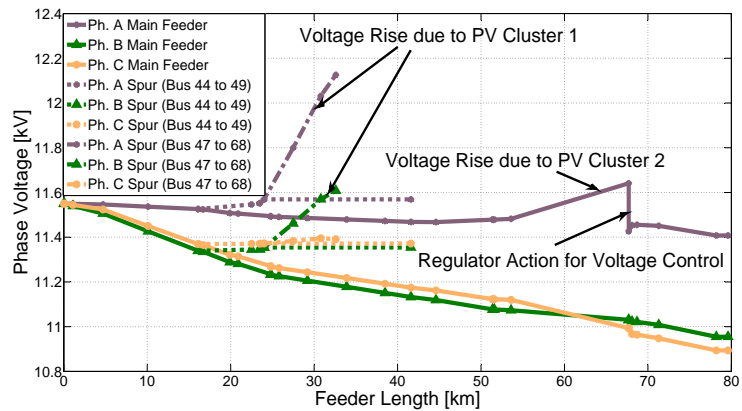

Fig. 11. Midday voltage profile along the $11 \mathrm{kV}$ feeder

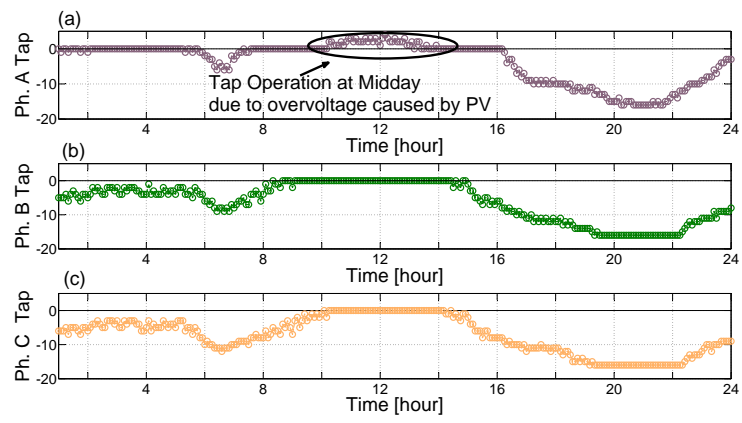

Fig. 12. Daily tap operations of REG3 (a) phase $a$ tap (b) phase $b$ tap (c) phase $c$ tap

downstream; phase $b$ and $c$ however, remains mostly at the neutral state as voltage in these phases remain within the limits. In the evening, tap numbers in all the phases are negative indicating a voltage boosting operation.

\section{CONCLUSiOnS}

A three-phase power flow approach capable of distribution network analysis by preserving the actual wiring configuration of MV and LV networks has been proposed for realistic analysis of solar PV impacts. This approach would be particularly useful for multigrounded LV networks where the neutral wire needs to be modeled explicitly due to the presence of neutral grounding impedances. A real distribution network, consisting of 3-wire MV and 4-wire LV feeders with multiple neutral groundings, has been used to verify the applicability of the proposed power flow approach. Unlike the the traditional 3wire power flow, the proposed approach is able to provide the neutral current and voltage caused by unbalanced allocation of single-phase rooftop PV units, and hence would be able to provide information regarding possible safety issues. With the peak level of PV output during midday, the neutral current and voltage impacts become more significant, as verified through a series of power flow calculations corresponding to a 24 hour load and PV output variations. Simultaneous modeling of 3-wire and 4-wire systems using the proposed approach enables the investigation the PV impacts propagated to the upstream MV level. Further research can be carried out using the proposed approach to investigate the voltage regulation coordination and protection issues against high neutral to ground voltage related to 3-wire MV and 4-wire multigrounded LV systems with distributed resources.

\section{ACKNOWLEDGMENT}

The authors gratefully acknowledge the support and cooperation of Essential Energy personnel for providing practical information and data on distribution networks and solar PV generation.

\section{REFERENCES}

[1] RA Walling, R. Saint, R.C. Dugan, J. Burke, and L.A. Kojovic, "Summary of distributed resources impact on power delivery systems", Power Delivery, IEEE Transactions on, vol. 23, no. 3, pp. 1636-1644, 2008.

[2] F. Katiraei, K. Mauch, and L. Dignard-Bailey, "Integration of photovoltaic power systems in high-penetration clusters for distribution networks and mini-grids", International Journal of Distributed Energy Resources, vol. 3, no. 3, pp. 207-224, 2007.

[3] M. Thomson and D.G. Infield, "Network power-flow analysis for a high penetration of distributed generation", Power Systems, IEEE Transactions on, vol. 22, no. 3, pp. $1157-1162$, aug. 2007.

[4] A. Canova, L. Giaccone, F. Spertino, and M. Tartaglia, "Electrical impact of photovoltaic plant in distributed network", Industry Applications, IEEE Transactions on, vol. 45, no. 1, pp. 341-347, 2009.

[5] A. von Jouanne and B. Banerjee, "Assessment of voltage unbalance", Power Delivery, IEEE Transactions on, vol. 16, no. 4, pp. $782-790$, oct 2001.

[6] P. Juanuwattanakul and M.A.S. Masoum, "Voltage stability enhancement for unbalanced multiphase distribution networks", in Power and Energy Society General Meeting, 2011 IEEE, july 2011, pp. 1 -6.

[7] Tsai-Hsiang Chen and Wen-Chih Yang, "Analysis of multi-grounded four-wire distribution systems considering the neutral grounding", Power Delivery, IEEE Transactions on, vol. 16, no. 4, pp. $710-717$, oct 2001.

[8] "Ieee recommended practice for grounding of industrial and commercial power systems", IEEE Std 142-2007 (Revision of IEEE Std 142-1991), pp. c1 -215, 302007.

[9] Ausgrid, "Design standards for distribution earthing", Available:http://www.ausgrid.com.au/ /media/Files/Network/ Documents/NS $\backslash \% 20$ and $\backslash \% 20 N U S / N S 116 \_N S A . p d f, \quad 2005, \quad J u n$, [Online].

[10] Ergon Energy, "Distribution system earthing guidelines", Available:http://www.ergon.com.au/_data/assets/pdf_file/0018/6615/ Distribution-System-Earthing-Guidelines.pdf, [Online].

[11] R.M. Ciric, A.P. Feltrin, and L.F. Ochoa, "Power flow in fourwire distribution networks-general approach", Power Systems, IEEE Transactions on, vol. 18, no. 4, pp. 1283-1290, 2003.

[12] JC Balda, AR Oliva, DW McNabb, and RD Richardson, "Measurements of neutral currents and voltages on a distribution feeder", Power Delivery, IEEE Transactions on, vol. 12, no. 4, pp. 1799-1804, 1997.

[13] T.M. Gruzs, "A survey of neutral currents in three-phase computer power systems", Industry Applications, IEEE Transactions on, vol. 26, no. 4, pp. 719-725, 1990.

[14] Power \& Systems Innovations Inc., "Power quality - the basics", Available:http://www.psihq.com/iread/pqbasics.htm, [Online].

[15] J.A. Martinez and J. Mahseredjian, "Load flow calculations in distribution systems with distributed resources. a review", in Power and Energy Society General Meeting, 2011 IEEE, july 2011, pp. 1 -8.

[16] P.A.N. Garcia, J.L.R. Pereira, Jr. Carneiro, S., V.M. da Costa, and N. Martins, "Three-phase power flow calculations using the current injection method", Power Systems, IEEE Transactions on, vol. 15, no. 2, pp. $508-514$, may 2000.

[17] D.R.R. Penido, L.R. de Araujo, S. Carneiro, J.L.R. Pereira, and P.A.N Garcia, "Three-phase power flow based on four-conductor current injection method for unbalanced distribution networks", Power Systems, IEEE Transactions on, vol. 23, no. 2, pp. 494 -503, may 2008.

[18] R. C. Dugan and S. Santoso, "An example of 3-phase transformer modeling for distribution system analysis", in Transmission and Distribution Conference and Exposition, 2003 IEEE PES, vol. 3, pp. 1028-1032 vol.3.

[19] W.H. Kersting, "Radial distribution test feeders", in Power Engineering Society Winter Meeting, 2001. IEEE. IEEE, 2001, vol. 2, pp. 908-912.

[20] J. Arrillaga and NR Watson, "Computer modelling of electric power systems (including facts)", 2001.

[21] N. Lu, Y. Xie, Z. Huang, F. Puyleart, and S. Yang, "Load component database of household appliances and small office equipment", in Power and Energy Society General Meeting-Conversion and Delivery of Electrical Energy in the 21st Century, 2008 IEEE. IEEE, 2008, pp. $1-5$. 
[22] K.P. Schneider and J.C. Fuller, "Detailed end use load modeling for distribution system analysis", in Power and Energy Society General Meeting, 2010 IEEE, july 2010, pp. 1 -7.

[23] G.M. Masters, Renewable and efficient electric power systems, WileyIEEE Press, 2004.

[24] M.G. Villalva, J.R. Gazoli, and E.R. Filho, "Comprehensive approach to modeling and simulation of photovoltaic arrays", Power Electronics, IEEE Transactions on, vol. 24, no. 5, pp. 1198 -1208, may 2009.

[25] Kyocera, "Kc200gt high efficiency multicrystal photovoltaic module", Available:http://www.kyocerasolar.com/assets/001/5195.pdf, [Online].

[26] J. Chan J. Werda and P. Freeman, "Electrical earthing-risk management strategies for the water industry", Available:http://www.wioa.org.au/ conference_papers/08_nsw/documents/JohnWerda.pdf, 2008, Apr, [Online].

\section{BIOGRAPHIES}

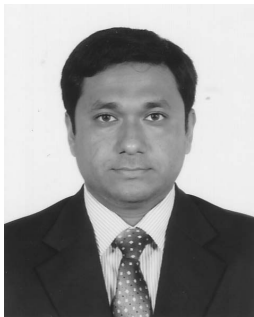

M J E Alam (Std. Member'10) received B.Sc. and M.Sc. Degree in Electrical and Electronic Engineering from Bangladesh University of Engineering and Technology, Dhaka, Bangladesh, in 2005 and 2009 , respectively, with focus on electrical energy and power systems.

At present he is conducting $\mathrm{PhD}$ research at the University of Wollongong, New South Wales, Australia. Prior to starting $\mathrm{PhD}$ studies, he has been involved with the power industry in Bangladesh for 4.5 years, where he worked in the field of power generation, transmission and distribution.

His research interest includes modeling and analysis of power systems considering the impacts of distributed and renewable energy resources.

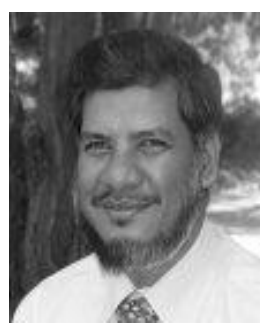

K M Muttaqi (M'01, SM'05) received the Ph.D degree from Multimedia University, Malaysia, in 2001. Currently, he is an Associate Professor at the School of Electrical, Computer, and Telecommunications Engineering, University of Wollongong, Wollongong, Australia. He was associated with the University of Tasmania, Australia as a Research Fellow/Lecturer/Senior Lecturer from 2002 to 2007 and with the Queensland University of Technology, Australia as a Research Fellow from 2000 to 2002. Previously, he also worked for Multimedia University as a Lecturer for three years. His special fields of interests include distributed generation, renewable energy, power system planning, intelligent grid, and power system reliability.

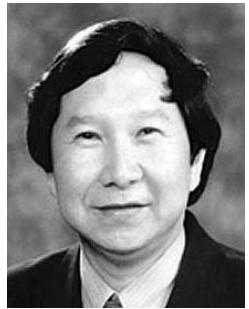

D Sutanto (SM'89) obtained his BEng. (Hons) and $\mathrm{PhD}$ from the University of Western Australia. He is presently the Professor of Power Engineering at the University of Wollongong, Australia. His research interests include power system planning, analysis and harmonics, FACTS and Battery Energy Storage systems. He is a Senior Member of IEEE. He is currently the IEEE IAS Area Chair for Region 10 (Asia Pacific). 